on to suggest that the class II-invariant chain complex is initially targeted to an early endocytic compartment and remains in the endocytic pathway until the class II molecule is released from invariant chain and binds an antigenic peptide $^{4}$. Our observation of mature class II molecules and invariant chain in both early and late endocytic compartments is compatible with dissociation being a gradual process and with the production of most functional antigenic complexes in later endocytic compartments, as proteolytic activity in the endocytic pathway increases. The class II-rich, lysosome-like endocytic compartment described by Peters et al. ${ }^{3}$ could be an endpoint for this progressive maturation of class II molecules, and perhaps the final compartment from which class II molecules can be released.

The discrepancy between our data and those of Peters et al. may be due to differences in techniques, cell lines or antibodies used, as suggested by Hackett. But the two studies addressed different questions. We induced formation of endocytic vesicles by internalization of crosslinked immunoglobulin (to mimic antigen uptake) and asked whether immature class II molecules could be found in this induced pathway, without focusing on the overall distribution of class II molecules in the cell, as addressed by Peters et al. In our subsequent analyses of the general distribution of class II molecules we have observed, in agreement with Peters et al., that most of the class II molecules are in the transGolgi reticulum of the cell, indicating a greater abundance in late endocytic compartments than in early endocytic compartments.

Nevertheless, we remain convinced of the specificity of our observed labelling of invariant chain-class II complexes in early endocytic compartments containing internalized immunoglobulin. In addition to the serological controls published in our original paper ${ }^{2}$, we have now documented that class I molecules in normal cells and invariant chains in class II-negative cells are not localized to such compartments ${ }^{4}$, demonstrating targeting specificity for the class II-invariant chain complex. Invariant chain has also been observed in early endosomes in melanoma cells (G. Griffiths, personal communication). Thus we favour a scheme of gradual maturation of class II molecules within the endocytic pathway which would allow them to acquire different antigenic fragments in different compartments, maximizing the reper-

1. Hackett, C. J. Nature 349, 655 (1991).

2. Guagliardi, L. E. et al. Nature 343, 133-139 (1990)

3. Peters, P. J., Neefjes, J. J., Oorschot, V., Ploegh, H. \& Geuze, H. J. Nature 349, 669676 (1991)

4. Brodsky, F. M. \& Guagliardi, L.E. A Rev. Immun. 9, 707-744 (1991). toire of peptides which can be bound and presented.

FRANCES M. BRODSKY

Departments of Pharmacy and

Pharmaceutical Chemistry,

School of Pharmacy,

University of California,

San Francisco,

California 94143-0446, USA

\section{Too exposed?}

SIR - We read with interest the Scientific Correspondence by D.K. Felbeck and W.H. Durrant (Nature 347, 341; 1990) reporting long strings of repetitive indentations from the surfaces of the long-duration exposure facility (LDEF) exposed to outer space. We have examined all components (clamps, experiment tray lips, intercostals and bolts) of the LDEF, searching for and characterizing features that could be caused by micrometeoroid and space debris impacts. We had previously observed the kinds of features reported by Felbeck and Durrant but had always thought them to be fabrication or handling artefacts. Although great care has been taken in handling LDEF nonexperiment components (such as the clamps described below and the experiand Durrant) following their return from orbit, handling damage could easily have occurred before flight.

As part of our responsibility as investigators with the LDEF meteoroid and debris special investigation group, we have been examining the chromicanodized aluminium (6061-T6) clamps which were used to hold experiment trays to the frame of the LDEF. The front face and the edges of these clamps were exposed to space for the entire duration of the 5.7-year orbital mission, but the back face of each clamp was not exposed to the orbital environment. We have now observed hundreds of linear, repetitive-type patterns, of the sort described by Felbeck and Durrant, on both the exposed and unexposed (back) ment tray lips characterized by Felbeck

faces of LDEF clamps. One such feature is shown in the figure. The occurrence of these features on the backs of clamps suggests that all such features are probably fabrication or handling defects, and not due to a previously unrecognized orbital exposure process. CECIL REDD

University of Houston at Clear Lake, Houston, Texas 77058, USA

SN2 NASA Johnson Space Center,

Houston, Texas 77058, USA

FELBECK REPLIES - We find that two important differences exist between Redd and Zolensky's observations and our own. First, the rough anodized surface of the tray clamps, as can be seen in their figure, severely limits resolution of fine details at high magnification in the electron microscope, in contrast to the remarkably smooth surface of the extruded aluminium-clad strips on which we made our observations. Second, the size of the repeat unit in their example, presumably typical of their observations, is of the order of 250 $\mu \mathrm{m}$, whereas the size of the repeat unit that we observed on the flight-exposed surfaces is of the order of $20-90 \mu \mathrm{m}$ in length.

We still find that the few repeat structures we have seen on the protected faces of the aluminium-clad strips are substantially less prevalent and much coarser than those on the flightexposed surfaces. We are planning a separate study of flight-exposed tray clamps from the LDEF, as well as of a non-flight control clamp. Even after careful examination of commercially available aluminium-clad material similar to our flight strips, we have not observed any repetitive structures of the sort observed on the flight-exposed surfaces from LDEF. In any event, a rational explanation for the source of these indentations is still lacking.

DAVID K. FELBECK

University of Michigan, Ann Arbor, Michigan 48109-2125, USA

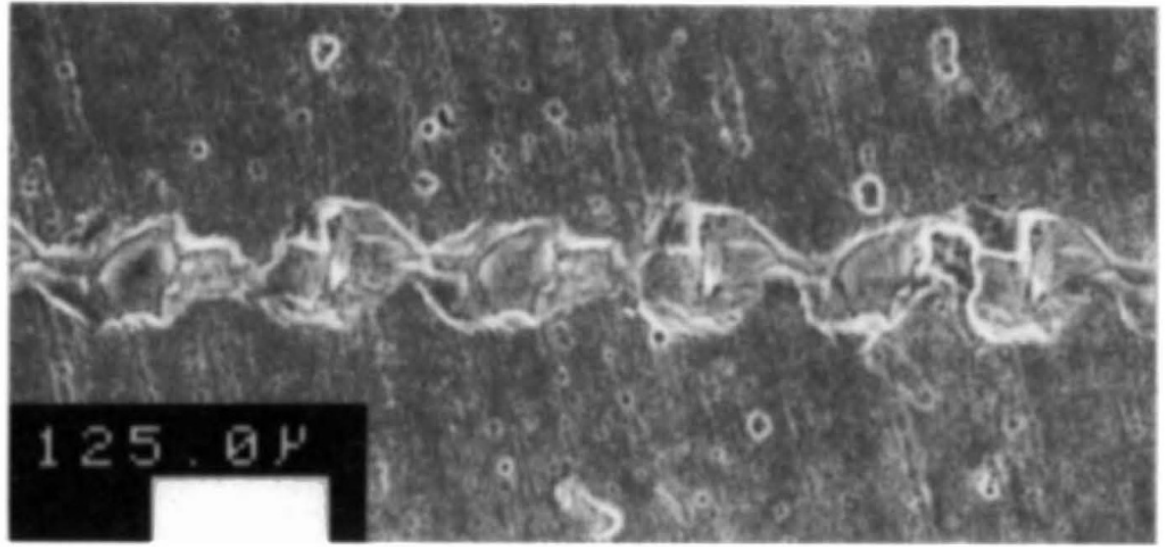

Scanning electron microscope image of a repetitive linear pattern found on the back (unexposed) side of an aluminium clamp from the LDEF satellite. Scale bar, $125 \mu \mathrm{m}$. 